\title{
Theoretical investigation on switchable second-order nonlinear optical (NLO) properties of novel cyclopentadienylcobalt linear [4]phenylene complexes
}

\author{
Wen-Yong Wang $\cdot$ Xiao-Feng Du $•$ Na-Na Ma $\cdot$ Shi-Ling Sun • \\ Yong-Qing Qiu
}

Received: 6 July 2012 / Accepted: 5 November 2012 / Published online: 11 January 2013

(C) The Author(s) 2013. This article is published with open access at Springerlink.com

\begin{abstract}
As a kind of novel organometallic complexes, the cyclopentadienylcobalt ( $\mathrm{CpCo})$ linear [4]phenylene complexes ( 4 = number of benzene rings) display efficient switchable nonlinear optical (NLO) response when $\mathrm{CpCo}$ reversibly migrates along the linear [4]phenylene triggered by heating or lighting. In this paper, the second-order NLO properties for $\mathrm{CpCo}$ linear [4]phenylene complexes were calculated by using the density functional theory (DFT) methods with four functionals. All of the functionals yield the same order of $\beta_{t o t}$ values: $1<2<4<3$. The effect of solvent on second-order NLO properties has been studied using polarized continuum model (PCM) in the tetrahydrofuran (THF) solution. The solvent leads to a slight enhancement of the NLO responses for the studied complexes relevant to their NLO responses in vacuo. The electronic absorption spectra were investigated by the TDDFT methods. The TDDFT calculations indicate that the maximum absorption peaks of complexes $2-4$ in the near-infrared spectrum area show the bathochromic shift together with a decreasing intensity compared to complex 1 . We have also found that the cobalt $(\mathrm{Co})$ atom acts as a donor in all the organometallic complexes and the $\mathrm{d} \rightarrow \pi^{*}$ and $\pi \rightarrow \pi^{*}$ charge transfer (CT) transitions contribute to the enhancement of second-order NLO response. Furthermore, two experimentally existing complexes 1 and 3 are found to have a large difference in $\beta_{t o t}$ values. It is our expectation that this difference may stimulate the search for a new type of switchable NLO material based on $\mathrm{CpCo}$ linear [4]phenylene complexes.
\end{abstract}

W.-Y. Wang $\cdot$ X.-F. Du $\cdot$ N.-N. Ma $\cdot$ S.-L. Sun $(\bowtie) \cdot$

Y.-Q. Qiu $(\bowtie)$

Institute of Functional Material Chemistry, Faculty of Chemistry,

Northeast Normal University, Changchun 130024,

People's Republic of China

e-mail: suns1430@nenu.edu.cn

e-mail: qiuyq466@nenu.edu.cn
Keywords CpCo linear [4]phenylene complex · DFT . Frequency dependent $\cdot$ NLO property

\section{Introduction}

Nonlinear optical (NLO) materials have been developed rapidly due to their potential utility in optical data storage, optical communication, optical computing, biological imaging, signal processing, and image reconstruction technologies [1-6]. Great efforts have been devoted to obtain highly efficient NLO materials. It is well-known that the molecular second-order NLO properties can be manipulated by modifying the donor and acceptor capacity and extending the $\pi$-conjugated bridge. Lots of work has been focused on push-pull molecules containing a donor and an acceptor connected via a $\pi$-conjugated bridge (D- $\pi-A)$ [7-9]. Generally, highly efficient NLO materials are mostly consistent with obvious charge transfer (CT) transitions. Thus, in most cases, the D- $\pi$-A structure is designed to enhance the CT transitions.

NLO activity can be found in organic compounds and inorganic crystals such as $\mathrm{LiNbO}_{3}$, and also in organometallic complexes $[10,11]$. Organic materials have lowenergy transitions in the UV-vis region which enhanced the NLO efficiency, but result in a tradeoff between nonlinear efficiency and optical transparency and they may have low thermal stability [12]. Inorganic crystals have several drawbacks: high quality single crystals are difficult to grow, are expensive, and are not easy to incorporate into electronic devices [11]. However, organometallic complexes offer greater scope for creation of multifunctional NLO materials by virtue of their greater design flexibility, low energy, and intense electronic transitions [12-14]. Since the 1980s, the investigations of the NLO properties for organometallic 
complexes have been especially arresting [15]. Those research works find that organometallic complexes can possess large NLO responses due to their lower transition energies and more intense CT transitions. Further, since the metal acts variously as a donor or an acceptor in the organometallic complexes, CT transitions of the organometallic complexes can be involved in three types: metal-toligand charge transfer (MLCT), ligand-to-metal charge transfer (LMCT) and metal-to-metal/intervalence charge transfer (MM/IVCT) [16]. In most cases, organometallic complexes have the noncentrosymmetric structure which is a universal requirement for the large first hyperpolarizability $(\beta)$. The $\beta$ value is often taken as a reference to establish NLO behavior.

Organometallic complexes also have the ability to switch their NLO properties through redox, deprotonation, turn onoff the conjugation, tautomerization reaction and so on [17, 18]. To reach the switchable characteristics, organometallic complexes must have two forms whose physical properties (or the $\beta$ values) are significantly different, and their thermal and chemical properties are both stable [19]. The design of organometallic complexes with high switchable NLO responses has motivated a lot of experimental works. Coe et al. used hyper-rayleigh scattering (HRS) and electronic stark effect (electroabsorption) spectroscopic measurements to probe the quadratic NLO effects of a range of ruthenium complexes $[14,20]$. Reversible $\mathrm{Ru}^{\mathrm{II} / \mathrm{III}}$ complexes are potentially redox-switchable chromophores [10, 21, 22]. Theoretical studies have also been performed to rationalize the switchable NLO properties. Liu has forecasted the switchable NLO properties of tetrathiafulvalene (TTF) derivatives by FF method [17, 23]. Theoretical work has enough ability to interpret the NLO property, even to design complexes with markedly switchable NLO responses in advance of experimental investigation.
In this paper, we investigated the $\mathrm{CpCo}$ linear [4]phenylene complexes which display efficient switchable NLO properties. Vollhardt et al. first reported the $\mathrm{CpCo}$ linear [4]phenylene complexes, in which $\mathrm{CpCo}$ undergos thermally reversible photometallahaptotropism along the linear [4] phenylene between the inner and outer cyclobutadiene ring (1 33 , see Fig. 1) [24]. To establish the path of $\mathrm{CpCo}$ migration, Vollhardt also computed the CpCo linear [4] phenylene complex 2 (whose $\mathrm{CpCo}$ is above the inner benzene ring). The $\mathrm{CpCo}$ linear [4]phenylene complexes may display switchable NLO properties, because the CpCo locates above the different cyclobutadiene and benzene rings. Nevertheless, a detailed understanding of the switchable NLO properties for the CpCo linear [4]phenylene complexes are still lacking. Hence, to better predict the switchable NLO responses of the $\mathrm{CpCo}$ linear [4]phenylene complexes, we designed complex 4 (whose $\mathrm{CpCo}$ is above the outer benzene ring) and studied the NLO properties of complexes 1,2,3 and 4. We hope this study may evoke the possibility to explore a new thriving area, i.e., CpCo linear [4]phenylene complexes for NLO application.

\section{Computational details}

The geometrical structures of CpCo linear [4]phenylene complexes 1-4 and linear [4]phenylene (See Fig. 2) were obtained by density functional theory (DFT) method at B3LYP/6-31G (d) (LanL2DZ basis set for Co ion) level with real frequency. The B3LYP functional reproduces the geometries of molecules containing transitional metals very well $[25,26]$. To obtain more accurate geometry, solvent effect has been taken into account in optimization and modeled using the polarized continuum model (PCM). We also optimized the geometrical structure of complex 1 in the tetrahydrofuran (THF) solution.
Fig. 1 Chemical structures for complexes 1-4 and linear [4]phenylene

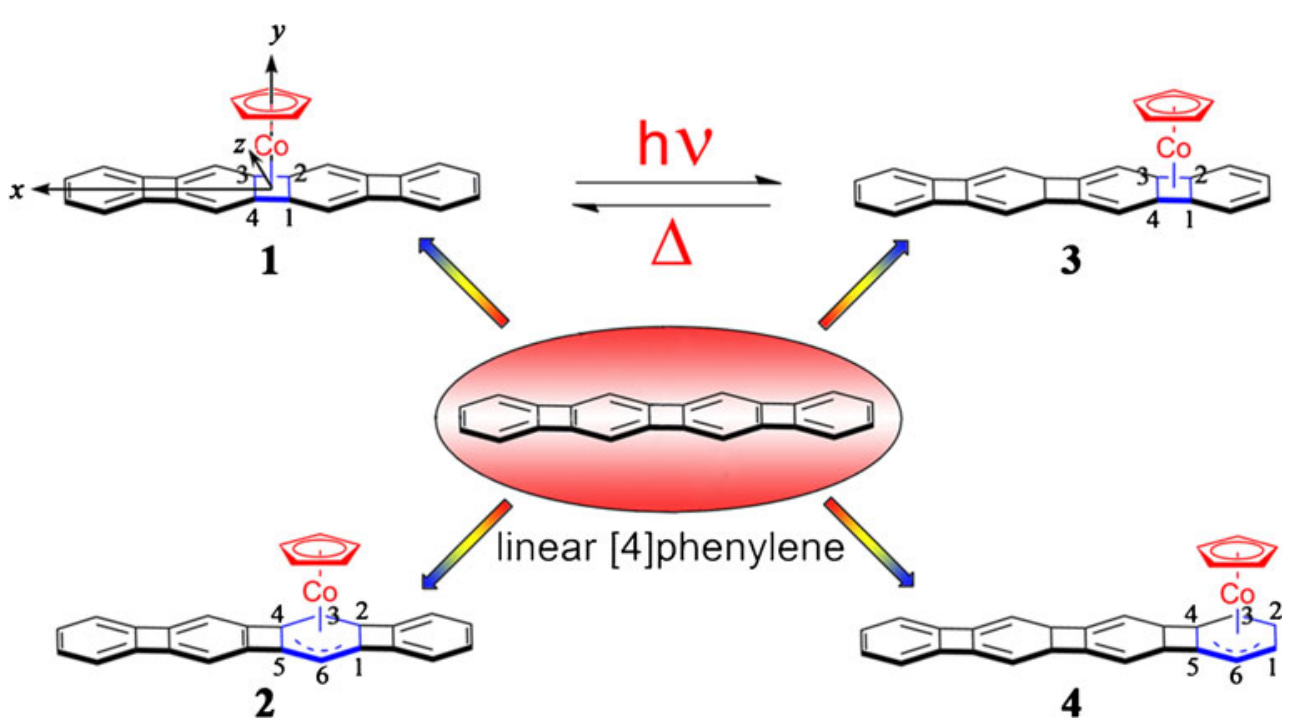


Fig. 2 Optimized structures for complexes 1-4 (side view) and linear [4]phenylene

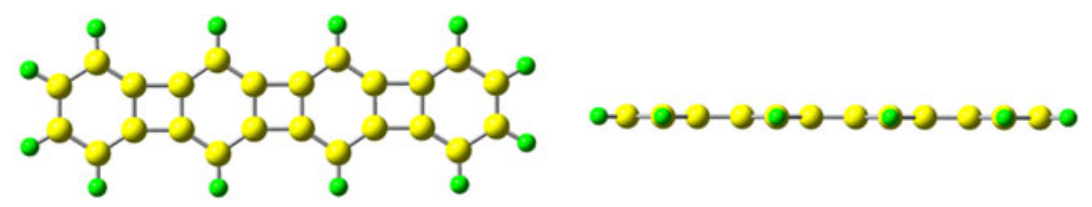

linear [4]phenylene (top view) linear [4]phenylene (side view)

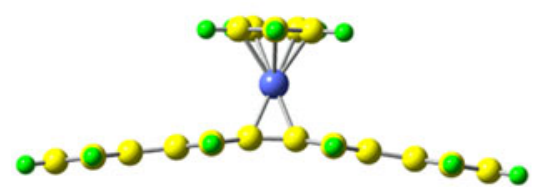

1

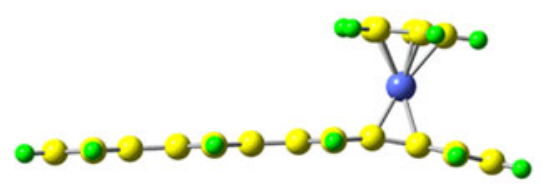

3
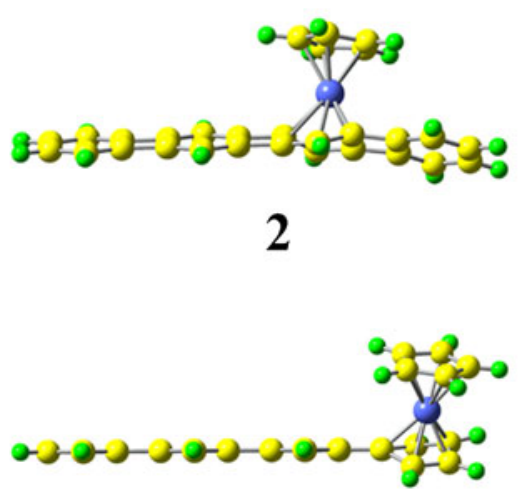

4
The computed results (the Co-C bond lengths only increase $\sim 0.002 \AA$ ) give very similar results to the vacuo calculations. This indicates that solvent effect on the geometrical structure is slight. Moreover, the calculation of natural bond orbital (NBO) analysis was performed at the B3LYP/6-31G(d) (LanL2DZ basis set for Co ion) level.

When a molecule is set in a uniform electric field $F$, its energy $E$ can be described by the following formula:

$$
\begin{aligned}
E(F)= & E(0)-\mu_{i} F_{i}-\frac{1}{2} \alpha_{i j} F_{i} F_{j}-\frac{1}{6} \beta_{i j k} F_{i} F_{j} F_{k} \\
& -\frac{1}{24} \gamma_{i j k l} F_{i} F_{j} F_{k} F_{l}-\ldots . .
\end{aligned}
$$

Where $\alpha_{i j}, \beta_{i j k}$ and $\gamma_{i j k l}$ are the polarizability, the first hyperpolarizability and second hyperpolarizability tensors, respectively. The subscripts $i, j$ and $k$ label $x, y$ and $z$ components. It is clear that the values of $\alpha_{i j}, \beta_{i j k}$ and $\gamma_{i j k l}$ can be obtained by differentiating $E$ with respect to $F$. In this work, the static first hyperpolarizability $\beta$ was calculated by analytical third energy derivatives, which is more efficient and less expensive [27]. The total second-order polarizabilities $\left(\beta_{t o t}\right)$ for the studied complexes are defined as:

$\beta_{\text {tot }}=\left(\beta_{x}^{2}+\beta_{y}^{2}+\beta_{z}^{2}\right)^{1 / 2}$,

Where $\beta_{i}$ is defined as:

$$
\beta_{i}=\left(\beta_{i i i}+\beta_{i j j}+\beta_{i k k}\right) \quad i, j, k=x, y, z .
$$

To check the consistency of our calculation, the $\beta$ value was calculated using Becke3-Lee-Yang-Parr (B3LYP) functional, long-range corrected (CAM-B3LYP) functional [28], half-andhalf (BHandHLYP) functional [29] and new hybrid meta (M06-2X) functinal [30] at 6-31++G(d) (LanL2DZ basis set for Co ion) level. It is clearly seen that the four functionals display the same trend in $\beta$ values. For clarity, we only take the CAM-B3LYP functional as an example to shed light on the changes in the first hyperpolarizabilities of the studied complexes. Furthermore, to understand the influence of the dispersion (frequency dependence) and the effect of the electron correlation on the NLO properties, the frequency-dependent $\beta$ of $\mathrm{CpCo}$ linear [4]phenylene complexes were evaluated using couple-perturbed (CP) DFT method with CAMB3LYP functional. In addition, the PCM model has been used to examine the solvent effect on the first static hyperpolarizabilities and frequency-dependent hyperpolarizabilities.

To further explain the second-order NLO behavior for the series of complexes, we employed time-dependent density functional theory (TDDFT) methods to descript their electronic spectra. TDDFT is one of the most accurate approaches for calculating the low-lying single excitations [31-33]. To choose suitable calculated methods, the electron absorption spectrum of complex 1 was simulated using TD-B3LYP, TDCAM-B3LYP, TD-BHandHLYP, TD-M06-2X and TD-BP86 functionals with $6-31++\mathrm{G}(\mathrm{d})$ (LanL2DZ basis set for Co ion) associated with PCM in THF solution. The maximum absorption peaks obtained by B3LYP, CAM-B3LYP, BHandHLYP, M06-2X and BP86 are $48 \mathrm{~nm}, 70 \mathrm{~nm}, 67 \mathrm{~nm}, 62 \mathrm{~nm}$ and $1 \mathrm{~nm}$ shorter than that of experimental data $(385 \mathrm{~nm})$, respectively. These results show that the absorption spectrum obtained by BP86 functional is in more reasonable agreement with the experimental data than other functionals. Also, to consider the 
solvent effects in absorption spectra, the absorption spectrum of complex 1 predicted by vacuo calculation has been performed on optimized geometry. The absorption spectrum of complex 1 predicted by vacuo calculation shows the blue shift (about $12 \mathrm{~nm}$ ) compared to the PCM calculation. Therefore, the effect of the PCM on the absorption spectrum is strong and the absorption spectra for all studied complexes were calculated by using the TD-BP86 functional in THF solution.

All of the calculations in this work were carried out by using the Gaussian $09 \mathrm{~W}$ program package [34]. The molecular orbital compositions were analyzed using the AOMIX $6.52[35,36]$ program package. We divided linear [4]phenylene and $\mathrm{CpCo}$ into two fragments.

\section{Results and discussion}

Geometrical structure and natural bond orbital (NBO) analysis

It is well-known that the geometrical structure has a great effect on the properties of organometallic complexes. For our investigation herein, the origin of Cartesian coordinate system was located at the middle of the linear [4]phenylenes for all the studied complexes and the linear [4]phenylenes were placed in the $x z$-plane with longitudinal axis along the $x$-direction, while $y$-axis pointed to the Co ion and is orthogonal to cyclopentadienyl (see Fig. 1), and all the geometrical structures with real frequencies were obtained at the B3LYP/6-31G(d) (LanL2DZ basis set for Co ion) level. The results show that the energy of complex 1 is $16.7 \mathrm{kcalmol}^{-1}$ more stable than that of complex 2 and is $6.6 \mathrm{kcalmol}^{-1}$ more stable than that of complex 3, which is similar to the work of Vollhardt et al. (16.1 kcalmol ${ }^{-1}$ and $7.6 \mathrm{kcalmol}^{-1}$, respectively). It is suggested that the optimized geometrical structures are reliable. From Fig. 2, it is clearly seen that the incorporation of $\mathrm{CpCo}$ has a significant influence on geometries of $\mathrm{CpCo}$ linear [4]phenylene complexes. In the optimized structures, we find that the structures of complexes 1 and 3 are bow-shaped with respect to linear [4]phenylene. Meanwhile, the structures of complexes 2 and 4 are twisted. This means that the geometrical structures of $\mathrm{CpCo}$ linear [4]phenylene complexes are different when $\mathrm{CpCo}$ is above the cyclopentadienyl and the benzene ring. In order to further understand the geometrical structures of $\mathrm{CpCo}$ linear [4]phenylene complexes, the selected bond lengths of Co-C between $\mathrm{CpCo}$ and linear [4]phenylene have been listed in Table 1 . The bond length of 3 is the shortest among complexes $1-4$, and the order of $\mathrm{Co}-\mathrm{C}$ is: $3>1>2>4$. This order demonstrates that various location of the $\mathrm{CpCo}$ remarkably affects the bond length.

To interpret the bond character and the interaction between $\mathrm{CpCo}$ and linear [4]phenylene, natural bond orbital (NBO) analysis has been taken into account, and the results have also been shown in Table 1. It can be found that the bond of $\mathrm{Co}-\mathrm{C}$ is formed by $\mathrm{p}$ orbital of $\mathrm{C}$ atom and $\mathrm{d}$ orbital of Co atom. The NBO charge of Co atoms for all complexes is about 0.5 a.u., and that of the linear [4]phenylene is about -0.2 a.u., as shown in Table 2. Therefore, we forecast that the CTs mainly occur between $\mathrm{Co}$ atom and linear [4]phenylene for all complexes.

\section{Electronic structure and absorption spectrum}

In order to obtain a more intuitive description of the band assignments of the electronic absorption spectra and the trends in the NLO behaviors of the studied complexes, TDDFT method has been performed on the excited states. The TDDFT calculated wavelengths $(\lambda, \mathrm{nm})$, excited state transition energies $\left(\Delta E_{g m}, \mathrm{eV}\right)$, oscillator strengths $\left(f_{o s}\right)$, and major molecular orbital transitions of complexes $1-4$ have been summarized in Table 3. The absorption spectra for complexes 1-4 have been plotted in Fig. 3. Complex 1 has a very strong maximum absorption peak at $385 \mathrm{~nm}$. It is also noteworthy that the maximum absorption peaks of complexes 2-4 show the bathochromic shift together with a decreasing intensity compared to complex 1 . Combined with Table 3, we can see the bathochromic shift of complex 2 is $11 \mathrm{~nm}$, and a relatively larger bathochromic shift of complex 3 and complex 4 is $75 \mathrm{~nm}$ and $71 \mathrm{~nm}$, respectively.

The first excited states of complexes 1-4 are generated by one-electron transfer from the highest occupied molecular orbital (HOMO) to the lowest unoccupied molecular orbital (LUMO). HOMO and LUMO are related to some
Table 1 Selected bond lengths $(\AA)$ and NBO analysis of complexes 1-4

\begin{tabular}{|c|c|c|c|c|}
\hline \multirow{2}{*}{$\begin{array}{l}\text { Complex } \\
1\end{array}$} & \multicolumn{2}{|c|}{ Bond length } & \multirow{2}{*}{$\frac{\text { Occupancy }}{1.6656}$} & \multirow{2}{*}{$\begin{array}{l}\text { Orbital coefficient } \\
0.6446 \mathrm{sp}^{34.91} \mathrm{~d}^{0.01}+0.7645 \mathrm{sp}^{0.03} \mathrm{~d}^{67.79}\end{array}$} \\
\hline & $\mathrm{C} 1-\mathrm{Co}$ & 2.019 & & \\
\hline & $\mathrm{C} 2-\mathrm{Co}$ & 2.022 & 1.6646 & $0.6446 \mathrm{sp}^{35.25} \mathrm{~d}^{0.01}+0.7645 \mathrm{sp}^{0.03} \mathrm{~d}^{67.25}$ \\
\hline \multirow[t]{2}{*}{2} & $\mathrm{C} 2-\mathrm{Co}$ & 2.090 & 1.7480 & $0.6720 \mathrm{sp}^{12.79} \mathrm{~d}^{0.00}+0.7405 \mathrm{sp}^{0.03} \mathrm{~d}^{73.55}$ \\
\hline & $\mathrm{C} 5-\mathrm{Co}$ & 2.113 & 1.7639 & $0.6849 \mathrm{sp}^{12.50} \mathrm{~d}^{0.00}+0.7286 \mathrm{sp}^{0.03} \mathrm{~d}^{58.72}$ \\
\hline \multirow[t]{2}{*}{3} & $\mathrm{C} 3-\mathrm{Co}$ & 1.999 & 1.7008 & $0.6583 \mathrm{sp}^{25.73} \mathrm{~d}^{0.01}+0.7527 \mathrm{sp}^{0.02} \mathrm{~d}^{42.91}$ \\
\hline & C4-Co & 2.000 & 1.7009 & $0.6585 \mathrm{sp}^{25.82} \mathrm{~d}^{0.01}+0.7526 \mathrm{sp}^{0.02} \mathrm{~d}^{42.93}$ \\
\hline 4 & $\mathrm{C} 3-\mathrm{Co}$ & 2.124 & 1.7710 & $0.6620 \mathrm{sp}^{17.63} \mathrm{~d}^{0.01}+0.7495 \mathrm{sp}^{0.06} \mathrm{~d}^{64.30}$ \\
\hline
\end{tabular}


Table 2 NBO charge of Co and linear [4]phenylene for complexes 1-4

\begin{tabular}{lrrrr}
\hline Charge & \multicolumn{1}{l}{2} & \multicolumn{1}{l}{3} & \multicolumn{1}{l}{4} \\
\hline Co & 0.5172 & 0.5583 & 0.5289 & 0.5354 \\
linear [4]phenylene & -0.2347 & -0.2813 & -0.2289 & -0.2287 \\
\hline
\end{tabular}

chemical properties of complexes such as redox and electronic transition, etc. The HOMOs and LUMOs have been plotted in Fig. 4. It can be found that the HOMO of complex 1 is localized on the whole molecule, but the HOMOs of complexes 2-4 are localized around the Co and cyclopentadienyl. We take $\mathrm{CpCo}$ and linear [4]phenylene as two fragments to consider each fragment MO contributions (\%) to the whole complex. The CpCo contribution to the HOMOs of complexes $1-4$ are $18.48 \%, 40.16 \%, 29.50 \%, 50.27 \%$, respectively. It suggests that $\mathrm{CpCo}$ acts as an important role in the $\mathrm{CpCo}$ linear [4]phenylene complexes. The LUMOs for complexes 1-3 are localized on the whole molecules. Whereas, the LUMO of complex 4 is localized on linear [4] phenylene and Co atom. However, oscillator strengths of the first excited states for the CpCo linear [4]phenylene complexes are small, which can be ignored. The crucial excited state with substantial oscillator strength is employed to observe the CT transitions clearly. As shown in Fig. 4, the major CT transition of complex 1 is from HOMO-5 to LUMO. The HOMO-5 of complex 1 is mainly localized on the linear [4]phenylene fragment and $\mathrm{Co}$ atom. The $\mathrm{MO}$ contribution of CpCo on HOMO-5 is $29.84 \%$, while the Co atom for MO contribution is $29.55 \%$. It implies that the significant CT transitions is mainly from Co atom to linear [4]phenylene and then to cyclopentadienyl. Specifically, these CT transitions are assigned to $\mathrm{d}(\mathrm{Co}) \rightarrow \pi^{*}$ (linear [4] phenylene) and $\pi$ (linear [4]phenylene) $\rightarrow \pi^{*}$ (linear [4]phenylene) transitions. The major CT transition of complex 2 is from HOMO to LUMO+5. The LUMO+5 of complex 2 is almost localized on the linear [4]phenylene fragment (99.00\%). This CT is assigned to $\mathrm{d}(\mathrm{Co}) \rightarrow \pi^{*}$ (linear [4] phenylene). The major CT of complex 3 is from HOMO-2 to

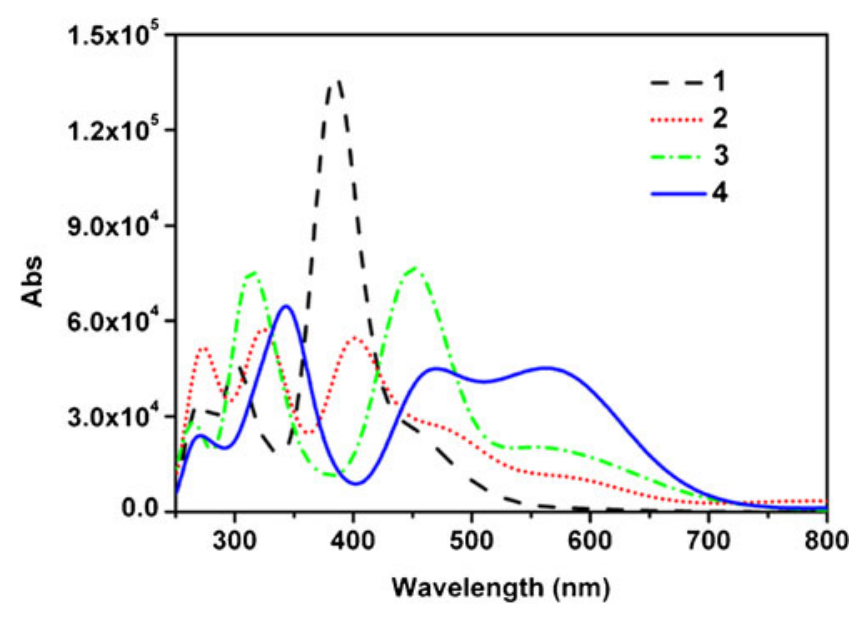

Fig. 3 Calculated optical spectra for complexes 1-4

LUMO+2. The HOMO-2 and LUMO+2 of complex 3 are almost localized on all the linear [4]phenylene fragment, too (73.36\% and $96.11 \%$, respectively). The major CT transition of complex 4 is from HOMO-1 to LUMO+2. HOMO-1 and LUMO+2 of complex 4 are similar to HOMO-2 and LUMO +2 of complex 3 , respectively. This illustrates that there are $\pi \rightarrow \pi^{*}$ transition in linear [4]phenylene and $d \rightarrow \pi^{*}$ transition between Co atom and linear [4]phenylene. And this is an important reason to produce second-order NLO response for all studied complexes.

\section{Second-order NLO property}

The calculated $\beta_{t o t}, \beta_{x}, \beta_{y}$ and $\beta_{z}$ values of complexes 1-4 at four different functionals have been listed in Table 4 and the variation tendency of $\beta_{t o t}$ values has been plotted in Fig. 5 . From Table 4, among complexes 2-4, the $\beta_{x}$ values dominate the second-order NLO response, because the skeleton atoms mostly locate on the $x$-axis, and the variation tendency of $\beta_{x}$ values for the studied complexes is similar to their $\beta_{\text {tot }}$ values. The $\beta_{y}$ values also significantly contribute to the second-order NLO responses. This indicates that CT transfer also occurs

Table 3 Wavelengths $(\lambda, \mathrm{nm})$, excited state transition energies $\left(\Delta E_{g e}, \mathrm{eV}\right)$, oscillator strengths $\left(f_{o s}\right)$, and major molecular orbital contributions of complexes 1-4 calculated using TDDFT method at the BP86/6-31++G(d) (LanL2DZ basis set for Co ion) level in the THF solution

\begin{tabular}{|c|c|c|c|c|c|}
\hline Complex & State & $\lambda$ & $\Delta E_{g e}$ & $f_{o s}$ & Major contributions (\%) \\
\hline \multirow[t]{2}{*}{1} & $\mathrm{~S}_{1}$ & 696 & 1.78 & 0.0000 & $\mathrm{HOMO} \rightarrow \mathrm{LUMO}(99 \%)$ \\
\hline & $\mathrm{S}_{20}$ & 385 & 3.22 & 1.7240 & HOMO-5 $\rightarrow$ LUMO(49 \%), HOMO-2 $\rightarrow$ LUMO+2(21\%) \\
\hline \multirow[t]{2}{*}{2} & $\mathrm{~S}_{1}$ & 855 & 1.45 & 0.0135 & $\mathrm{HOMO} \rightarrow \mathrm{LUMO}(82 \%)$ \\
\hline & $\mathrm{S}_{19}$ & 396 & 3.13 & 0.2910 & $\mathrm{HOMO} \rightarrow \mathrm{LUMO}(18 \%), \mathrm{HOMO}-3 \rightarrow \mathrm{LUMO}+3(16 \%)$ \\
\hline \multirow[t]{2}{*}{3} & $\mathrm{~S}_{1}$ & 771 & 1.61 & 0.0004 & $\mathrm{HOMO} \rightarrow \mathrm{LUMO}(97 \%)$ \\
\hline & $\mathrm{S}_{14}$ & 460 & 2.70 & 0.6737 & $\mathrm{HOMO}-2 \rightarrow \mathrm{LUMO}+2(54 \%), \mathrm{HOMO}-5 \rightarrow \mathrm{LUMO}(14 \%)$ \\
\hline \multirow[t]{2}{*}{4} & $\mathrm{~S}_{1}$ & 1001 & 1.24 & 0.0505 & $\mathrm{HOMO} \rightarrow \mathrm{LUMO}(92 \%)$ \\
\hline & $\mathrm{S}_{14}$ & 456 & 2.72 & 0.3829 & $\mathrm{HOMO}-1 \rightarrow \mathrm{LUMO}+2(38 \%), \mathrm{HOMO}-1 \rightarrow \mathrm{LUMO}+3(30 \%)$ \\
\hline
\end{tabular}


Fig. 4 Major molecular orbital transitions of complexes 1-4

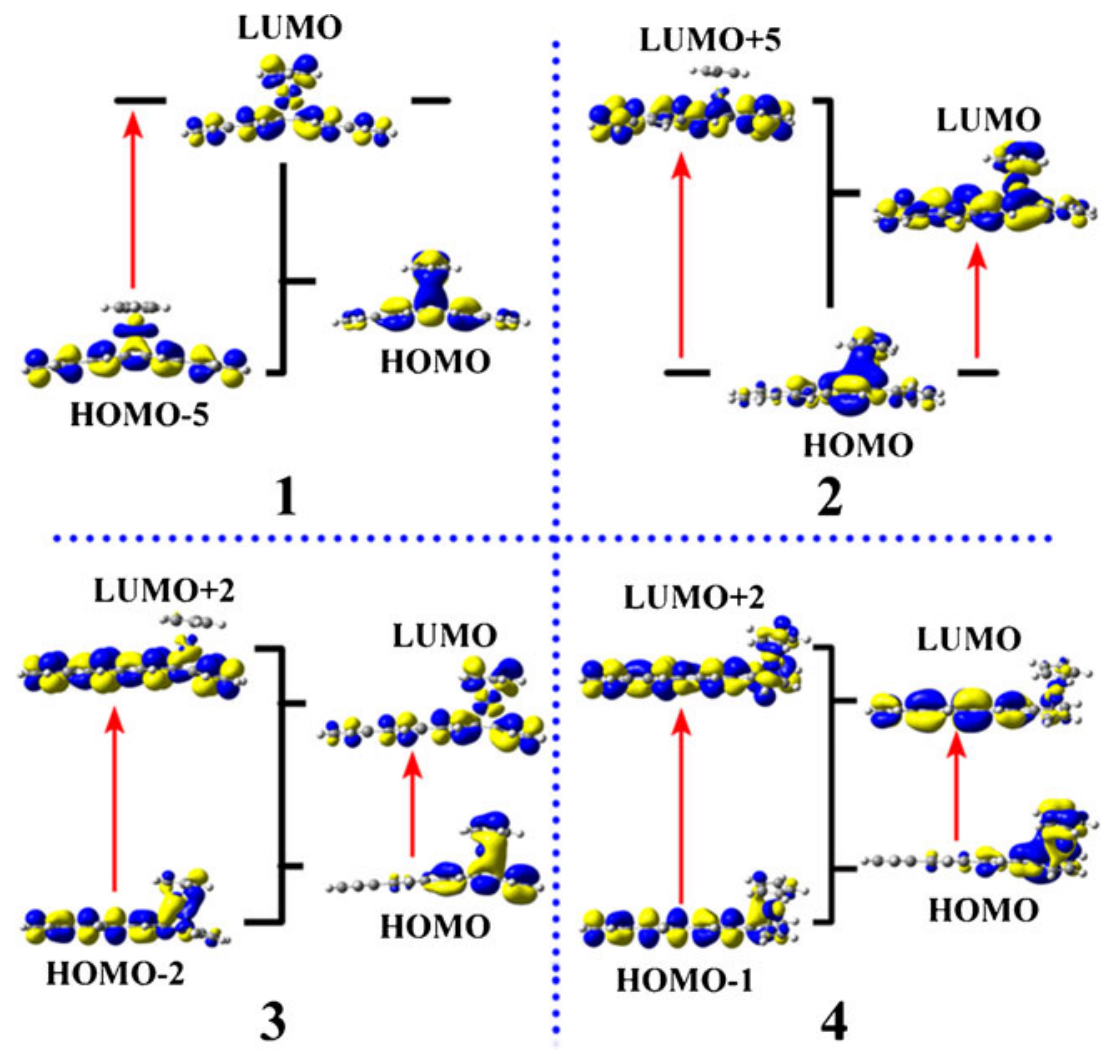

along $y$-axis. The $\beta_{x}$ value of complex 1 is close to zero. But why does the $\beta_{x}$ value of complex 1 become close to naught suddenly? To understand it, the structure-property relationship of complex 1 is taken into account. As we know, linear [4] phenylene is a centrosymmetric molecule. When the CoCp is in the middle of linear [4]phenylene, complex 1 is symmetric structure, and it is therefore sufficient for revealing the relationship between the $\beta_{x}$ value and the geometrical structure.

Table 4 The $\beta_{t o t}, \beta_{x}, \beta_{y}$ and $\beta_{z}$ values $\left(\times 10^{-30} \mathrm{esu}\right)$ for complexes 1-4 have been obtained by four methods. Effect of solvent on first-order hyperpolarizability has also been obtained by CAM-B3LYP method at the polarizable continuum model (PCM) in the presence of THF solution

\begin{tabular}{|c|c|c|c|c|c|}
\hline Complex & Method & $\beta_{x}$ & $\beta_{y}$ & $\beta_{z}$ & $\beta_{t o t}$ \\
\hline \multirow[t]{5}{*}{1} & CAM-B3LYP & -0.003 & -19.152 & 0.104 & 19.152 \\
\hline & CAM-B3LYP (PCM) & -0.014 & -48.413 & 0.291 & 48.414 \\
\hline & BHandHLYP & -0.005 & -23.094 & 0.067 & 23.094 \\
\hline & M06-2X & -0.004 & -25.601 & 0.111 & 25.602 \\
\hline & B3LYP & -0.002 & -22.655 & 0.100 & 22.655 \\
\hline \multirow[t]{5}{*}{2} & CAM-B3LYP & 18.691 & -13.953 & -7.539 & 24.513 \\
\hline & CAM-B3LYP (PCM) & 39.734 & -35.344 & -19.226 & 56.548 \\
\hline & BHandHLYP & 23.502 & -17.210 & -8.689 & 30.398 \\
\hline & M06-2X & 25.135 & -19.160 & -10.227 & 33.218 \\
\hline & B3LYP & 39.634 & -15.517 & -8.535 & 43.410 \\
\hline \multirow[t]{5}{*}{3} & CAM-B3LYP & 29.218 & -14.567 & -0.749 & 32.656 \\
\hline & CAM-B3LYP (PCM) & 59.950 & -37.132 & -1.583 & 70.535 \\
\hline & BHandHLYP & 43.045 & -18.970 & -1.121 & 47.053 \\
\hline & M06-2X & 43.664 & -21.010 & -1.159 & 48.470 \\
\hline & B3LYP & 58.468 & -15.624 & -1.433 & 60.536 \\
\hline \multirow[t]{5}{*}{4} & CAM-B3LYP & 24.098 & -11.533 & 6.134 & 27.411 \\
\hline & CAM-B3LYP (PCM) & 44.407 & -28.185 & 15.675 & 54.882 \\
\hline & BHandHLYP & 34.964 & -16.418 & 8.360 & 39.521 \\
\hline & M06-2X & 33.757 & -17.848 & 9.537 & 39.357 \\
\hline & B3LYP & 57.789 & -15.519 & 8.889 & 60.494 \\
\hline
\end{tabular}




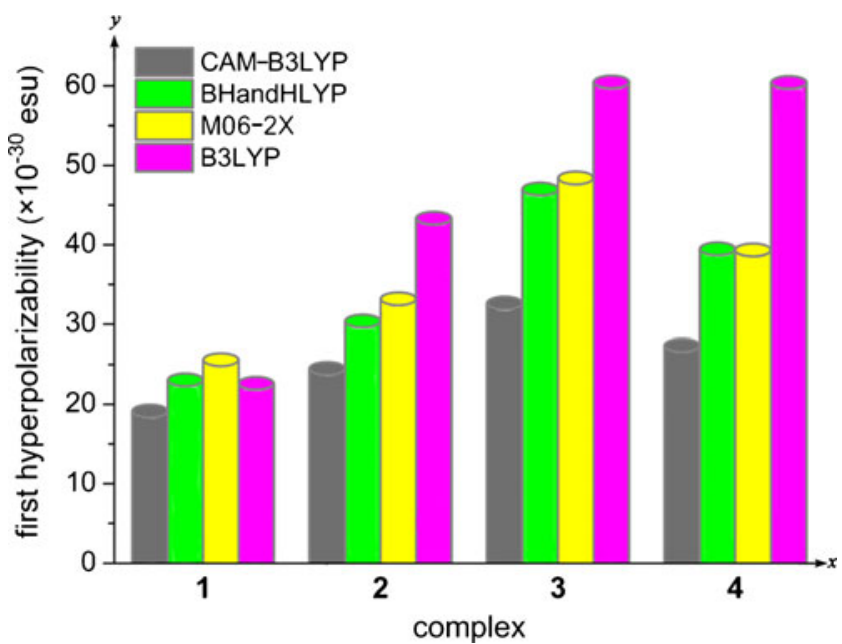

Fig. 5 The tendency of $\beta_{t o t}$ values for complexes 1-4

For CAM-B3LYP functional results, due to the change of CpCo location along the linear [4]phenylene, there is a stepwise escalation for the $\beta_{t o t}$ values of $\mathrm{CpCo}$ linear [4]phenylene complexes: $1<2<4<3$. It illustrates that the second order NLO responses could be tuned by changing the location of $\mathrm{CpCo}$. The solvent effect is also taken into account for the first hyperpolarizabilitiy. The static first hyperpolarizabilities are calculated in THF solvent by CAM-B3LYP functinonal (see Table 4). The results show that the hyperpolarizabilities are in the range of $\sim 2.0$ to $\sim 2.5$ times larger than that of calculations in vacuo. However, the order of hyperpolarizabilities for the studied complexes is still in accordance with the calculations in vacuo.

To have an insight into the origin of second-order NLO responses and explain the position effect of CpCo on NLO responses, we consider the widely used two-level model established by Oudar and Chemla [37]. The two-level model expression is defined as:

$\beta \propto \Delta \mu_{g m} f_{o s} / \Delta E_{g m}^{3}$,

Where $\Delta \mu_{g m}$ is the difference between excited and ground state dipole moments, $f_{o s}$ is the oscillator strength, $\Delta E_{g m}$ is the transition energy. From the expression, the $\beta$ value is inversely proportional to the cube of transition energy. Obviously, the transition energy is the decisive factor in the $\beta$ value.

The crucial transition energies of the four complexes were obtained by the TDDFT at pure functional BP86. The crucial transition energies show a descending trend for complexes 14: $1(3.22 \mathrm{eV})>2(3.13 \mathrm{eV})>4(2.72 \mathrm{eV})>3(2.70 \mathrm{eV})$, which is inversely proportional to the $\beta_{\text {tot }}$ values of $\mathrm{CpCo}$ linear [4] phenylene complexes $1-4$. In addition to this, the descending trend of crucial transition energies explains the gradually increase of $\beta_{t o t}$ values as the location of the $\mathrm{CpCo}$ changes. As a result, changing the position of $\mathrm{CpCo}$ can obtain a good NLO candidate.

Frequency-dependent second-order NLO properties

The frequency-dependent NLO properties of the CpCo linear [4]phenylene complexes are evaluated by couple-perturbed (CP) DFT method with CAM-B3LYP functional, and the same basis sets with static $\beta_{\text {tot }}$ value calculation are used. We calculated the frequency dependent first hyperpolarizability $\beta(-2 \omega ; \omega, \omega)$ for the second harmonic generation (SHG) and $\beta(-\omega ; \omega, 0)$ for the electro-optical Pockels effect (EOPE). It is very important to choose an appropriate frequency $\omega$ to calculate frequency-dependent NLO properties of these CpCo linear [4]phenylene complexes. Generally, the molecular hyperpolarizabilities, $\beta(-2 \omega ; \omega, \omega)$, have been measured in a fundamental incident wavelength which has a second harmonic far enough from the absorption bands to avoid the overmeasure of $\beta$ values due to resonance effects. Hence, two near resonant wavelength of $\omega=0.0428$ a.u. $(1064 \mathrm{~nm})$ and $\omega=0.0340$ a.u. $(1340 \mathrm{~nm})$, one nonresonant wavelength of $\omega=$ 0.0239 a.u. (1907 nm) are adopted to compute the frequencydependence. As shown in Table 5, the magnitude of the frequency-dependent first hyperpolarizability increases with the increasing frequency. The values of $\beta(-2 \omega ; \omega, \omega)$ in the CpCo linear [4]phenylene complexes are larger than that of $\beta(-\omega ; \omega, 0)$, and both of the $\beta(-2 \omega ; \omega, \omega)$ and $\beta(-\omega ; \omega, 0)$ values are larger than the corresponding static $\beta_{\text {tot }}$ values. Thus,
Table 5 The frequencydependent $\beta$ values $\left(\times 10^{-30}\right.$ esu) and effect of solvent (in the THF solution) on frequency-dependent $\beta$ values for complexes $1-4$ have been evaluated by coupleperturbed (CP) DFT method, CAM-B3LYP functional

a: The $\beta(-2 \omega ; \omega, \omega)$ value of complex 1 at $\omega=0.1300$ a.u. is $26716.925 \times 10^{-30}$ esu. b: The $\beta(-2 \omega ; \omega, \omega)$ value of complex 3 at $\omega=0.1300$ a.u. is $234770.958 \times 10^{-30} \mathrm{esu}$

\begin{tabular}{|c|c|c|c|c|c|c|}
\hline \multirow[t]{2}{*}{ Complex } & \multicolumn{2}{|c|}{ 0.0239(a.u.) } & \multicolumn{2}{|c|}{ 0.0340(a.u.) } & \multicolumn{2}{|c|}{ 0.0428(a.u.) } \\
\hline & $\beta(-\omega ; \omega, 0)$ & $\beta(-2 \omega ; \omega, \omega)$ & $\beta(-\omega ; \omega, 0)$ & $\beta(-2 \omega ; \omega, \omega)$ & $\beta(-\omega ; \omega, 0)$ & $\beta(-2 \omega ; \omega, \omega)$ \\
\hline $1^{\mathrm{a}}$ & 20.238 & 22.827 & 21.487 & 27.841 & 23.088 & 32.297 \\
\hline 1(PCM) & 37.664 & 35.799 & 40.197 & 44.306 & 43.472 & 52.602 \\
\hline 2 & 26.664 & 35.904 & 29.129 & 54.323 & 31.934 & 411.106 \\
\hline 2(PCM) & 46.130 & 52.757 & 51.004 & 84.878 & 57.066 & 704.092 \\
\hline $3^{\mathrm{b}}$ & 35.088 & 40.805 & 37.838 & 73.428 & 41.320 & 46.439 \\
\hline 3(PCM) & 57.703 & 58.558 & 62.563 & 119.442 & 68.782 & 71.459 \\
\hline 4 & 30.018 & 37.059 & 33.085 & 53.651 & 37.132 & 105.378 \\
\hline 4(PCM) & 47.106 & 51.893 & 52.408 & 77.772 & 59.560 & 164.025 \\
\hline
\end{tabular}


$\beta(-2 \omega ; \omega, \omega)$ exhibits the largest frequency dispersion. Since the switching between structures from complex 1 to complex 3 is induced by light at $\omega=0.1300$ a.u. ( $350 \mathrm{~nm}$ ), we also calculated the $\beta(-2 \omega ; \omega, \omega)$ values of complexes 1 and 3 at this wavelength. The $\beta(-2 \omega ; \omega, \omega)$ value of complex 3 is 8.8 times as large as that of complex 1 (see Table 5). The $\beta(-2 \omega ; \omega, \omega)$ values for complexes 1,3 at $\omega=0.1300$ a.u. and for complexes 2,4 at $\omega=0.0428$ a.u. are significantly enhanced, which could be caused by a resonant effect due to the weak absorption around second harmonic. Meanwhile, the frequencydependent hyperpolarizabilities in THF were calculated using CPDFT method with CAM-B3LYP functional for complexes 1-4. As shown in Table 4, the solvent also leads to a slight enhancement of the frequency-dependent hyperpolarizability for each complex relevant to its frequency-dependent hyperpolarizability in vacuo.

Switchable NLO response with larger $\beta$ value contrast

In this section, reversibly switchable NLO behaviors were investigated in detail. To obtain NLO switches, apart from the reversible regulating measure, their $\beta$ values contrast must be obvious. As discussed in "Geometrical structure and natural bond orbital (NBO) analysis" section, complexes 1 and 3 are more stable, which means that they are easier to be obtained and this explains why Vollhardt only got the CpCo linear [4]phenylene complexes 1 and 3 [24]. As listed in Table 4, the $\beta_{\text {tot }}$ value of complex 1 is $19.152 \times$ $10^{-30}$ esu calculated by CAM-B3LYP functional, while the value of complex 3 becomes larger $\left(32.656 \times 10^{-30} \mathrm{esu}\right)$. The $\beta_{t o t}$ value of complex 3 is larger, so we consider complex 3 as the "ON" state. Conversely, we forecast complex 1 as the "OFF" state. Therefore, the CpCo linear [4]phenylene complexes 1 and 3 display NLO switching properties when the CpCo migrates along the linear [4]phenylene between the inner and outer cyclobutadiene ring, and we believe that $\mathrm{CpCo}$ linear [4]phenylene complexes are promising in switchable NLO materials.

\section{Conclusions}

The geometrical structures and second-order NLO properties of $\mathrm{CpCo}$ linear [4]phenylene complexes were investigated by DFT methods. The theoretical investigation on geometrical structure suggests that the incorporation of $\mathrm{CpCo}$ gives rise to a significant distortion of planar linear [4]phenylene. Meanwhile, the NBO analysis demonstrates that the bond between $\mathrm{Co}$ and $\mathrm{C}$ atom is comprised of $\mathrm{p}$ orbital and d orbital, and the NBO charge demonstrates that CT occurs mainly from the Co atom to linear [4]phenylene. The electronic absorption spectra were investigated by the TDDFT methods. According to the TDDFT calculations, the $\mathrm{d} \rightarrow \pi^{*}$ and $\pi \rightarrow \pi^{*}$ CTs can contribute to enhance the secondorder NLO responses, and the order of the low transition energy for $\mathrm{CpCo}$ linear [4]phenylene complexes is inversely to their second-order NLO responses. Moreover, for the DFT results, due to the change of CpCo location along the linear [4] phenylene, there are large variations in the $\beta_{t o t}$ values of $\mathrm{CpCo}$ linear [4]phenylene complexes. The frequency-dependent first hyperpolarizabilities of the $\mathrm{CpCo}$ linear [4]phenylene complexes are also evaluated. From the results, $\beta(-2 \omega ; \omega, \omega)$ exhibits the largest frequency dispersion and the magnitude of the first hyperpolarizabilities increase with increasing frequency. In addition, the solvent leads to a slight enhancement of the hyperpolarizability and frequencydependent hyperpolarizability.

These studies propose a novel field, wherein the $\beta_{t o t}$ value can be changed obviously by migrating the $\mathrm{CpCo}$ between the inner and the outer cyclobutadiene rings. We hope this new type of switchable NLO material based on CpCo linear [4]phenylene complexes will be developed rapidly in the future.

Acknowledgments The authors gratefully acknowledge the financial support from the Natural Science Foundation of China (No. 21173035), the Natural Science Foundation of Jilin province (20101154).

Open Access This article is distributed under the terms of the Creative Commons Attribution License which permits any use, distribution, and reproduction in any medium, provided the original author(s) and the source are credited.

\section{References}

1. Marder SR (2006) Organic nonlinear optical materials: where we have been and where we are going. Chem Commun 131-134. doi: 10.1039/B512646K

2. Long NJ (1995) Organometallic compounds for nonlinear optics-The search for En-light-enment! Angew Chem Int Ed 34:21-38. doi:10.1002/anie.199500211

3. Kuzyk MG (2009) Using fundamental principles to understand and optimize nonlinear-optical materials. J Mate Chem 19:7444-7465. doi:10.1039/B907364G

4. Heck J, Dabek S, Meyer-Friedrichsen T, Wong H (1999) Monoand dinuclear sesquifulvalene complexes, organometallic materials with large nonlinear optical properties. Coord Chem Rev 190 192:1217-1254. doi:10.1016/S0010-8545(99)00177-0

5. Marder SR, Beratan DN, Cheng LT (1991) Approaches for optimizing the first electronic hyperpolarizability of conjugated organic molecules. Science 252:103-106. doi:10.1126/ science. 252.5002 .103

6. Zhang C, Song YL, Wang X (2007) Correlations between molecular structures and third-order non-linear optical functions of heterothiometallic clusters: a comparative study. Coord Chem Rev 251:111-141. doi:10.1016/j.ccr.2006.06.007

7. Green KA, Cifuentes MP, Samoc M, Humphrey MG (2011) Metal alkynyl complexes as switchable NLO systems. Coord Chem Rev 255:2530-2541. doi:10.1016/j.ccr.2011.02.021 
8. Asselberghs I, Clays K, Persoons A, Ward MD, McCleverty J (2004) Switching of molecular second-order polarisability in solution. J Mater Chem 14:2831-2839. doi:10.1039/B401434K

9. Ma NN, Liu CG, Qiu YQ, Sun SL, Su ZM (2012) Theoretical investigation on redox-switchable second-order nonlinear optical responses of push-pull $\mathrm{Cp} * \mathrm{CoEt} 2 \mathrm{C} 2 \mathrm{~B} 4 \mathrm{H} 3$-expanded (metallo)porphyrins. J Comput Chem 33:211-219. doi:10.1002/ jcc. 21966

10. Coe BJ (1999) Molecular materials possessing switchable quadratic nonlinear optical properties. Chem Eur J 5:2464-2471. doi:10.1002/ (sici)1521-3765(19990903)5:9<2464::aid-chem2464>3.0.co;2-1

11. Verbiest T, Houbrechts S, Kauranen M, Clays K, Persoons A (1997) Second-order nonlinear optical materials: recent advances in chromophore design. J Mater Chem 7:2175-2189. doi:10.1039/ A703434B

12. Powell CE, Humphrey MG (2004) Nonlinear optical properties of transition metal acetylides and their derivatives. Coord Chem Rev 248:725-756. doi:10.1016/j.ccr.2004.03.009

13. Barlow S, Marder SR (2000) Electronic and optical properties of conjugated group 8 metallocene derivatives. Chem Commun 15551562. doi:10.1039/b004907g

14. Coe BJ, Harris JA, Jones LA, Brunschwig BS, Song K, Clays K, Garín J, Orduna J, Coles SJ, Hursthouse MB (2005) Syntheses and properties of two-dimensional charged nonlinear optical chromophores incorporating redox-switchable cis-Tetraammineruthenium (II) centers. J Am Chem Soc 127:4845-4859. doi:10.1021/ ja0424124

15. Bella SD (2001) Second-order nonlinear optical properties of transition metal complexes. Chem Soc Rev 30:355-366. doi:10.1039/ B100820J

16. Coe BJ (2006) Switchable nonlinear optical metallochromophores with pyridinium electron acceptor groups. Acc Chem Res 39:383393. doi:10.1021/ar050225k

17. Liu CG, Su ZM, Guan XH, Muhammad S (2011) Redox and photoisomerization switching the second-order nonlinear optical properties of a tetrathiafulvalene derivative across six tStates: A DFT Study. J Phys Chem C 115:23946-23954. doi:10.1021/ jp2049958

18. Plaquet A, Guillaume M, Champagne B, Rougier L, Mancois F, Rodriguez V, Pozzo JL, Ducasse L, Castet F (2008) Investigation on the second-order nonlinear optical responses in the keto-enol equilibrium of anil derivatives. J Phys Chem C 112:5638-5645. doi:10.1021/jp711511t

19. Guan W, Yang GC, Liu CG, Song P, Fang L, Yan LK, Su ZM (2008) Reversible redox-switchable second-order optical nonlinearity in polyoxometalate: a quantum chemical study of $\left[\mathrm{PW}_{11} \mathrm{O}_{39}(\mathrm{ReN})\right]_{\mathrm{n}}{ }^{-}$ $(n=3-7)$. Inorg Chem 47:5245-5252. doi:10.1021/ic8001527

20. Coe BJ, Jones LA, Harris JA, Brunschwig BS, Asselberghs I, Clays K, Persoons A, Garín J, Orduna J (2004) Syntheses and spectroscopic and quadratic nonlinear optical properties of extended dipolar complexes with Ruthenium(II) ammine electron donor and N-Methylpyridinium acceptor groups. J Am Chem Soc 126:3880 3891. doi:10.1021/ja0315412

21. Coe BJ, Foxon SP, Harper EC, Helliwell M, Raftery J, Swanson CA, Brunschwig BS, Clays K, Franz E, Garìn J, Orduna JS, Horton PN, Hursthouse MB (2010) Evolution of linear absorption and nonlinear optical properties in V-shaped Ruthenium(II)-based chromophores. J Am Chem Soc 132:1706-1723. doi:10.1021/ja908667p

22. Coe BJ, Houbrechts S, Asselberghs I, Persoons A (1999) Efficient, reversible redox-switching of molecular first hyperpolarizabilities in Ruthenium(II) complexes possessing large quadratic optical nonlinearities. Angew Chem Int Ed 38:366-369. doi:10.1002/ (sici)1521-3773(19990201)38:3<366::aid-anie366>3.0.co;2-d

23. Liu CG, Guan XH, Su ZM (2011) Computational study on redoxswitchable 2D second-order nonlinear optical properties of push pull mono-tetrathiafulvalene- Bis(salicylaldiminato) Zn(II) Schiff base complexes. J Phys Chem C 115:6024-6032. doi:10.1021/ jp111797n

24. Albright TA, Oldenhof S, Oloba OA, Padilla R, Vollhardt KPC (2011) Reversible intercyclobutadiene haptotropism in cyclopentadienylcobalt linear [4]phenylene. Chem Commun 47:90399041. doi:10.1039/C1CC13405A

25. Becke AD (1993) Density-functional thermochemistry. III. The role of exact exchange. J Chem Phys 98:5648-5652. doi:10.1063/ 1.464913

26. Ivakhnenko EP, Starikov AG, Minkin VI, Lyssenko KA, Antipin MY, Simakov VI, Korobov MS, Borodkin GS, Knyazev PA (2011) Synthesis, molecular and electronic structures of six-coordinate transition metal $(\mathrm{Mn}, \mathrm{Fe}, \mathrm{Co}, \mathrm{Ni}, \mathrm{Cu}$, and $\mathrm{Zn}$ ) complexes with redox-active 9-hydroxyphenoxazin-1-one ligands. Inorg Chem 50:7022-7032. doi:10.1021/ic2004062

27. Chopra P, Carlacci L, King HF, Prasad PN (1989) Ab initio calculations of polarizabilities and second hyperpolarizabilities in organic molecules with extended.pi.-electron conjugation. J Phys Chem 93:7120-7130. doi:10.1021/j100357a020

28. Yanai T, Tew DP, Handy NC (2004) A new hybrid exchangecorrelation functional using the Coulomb-attenuating method (CAM-B3LYP). Chem Phys Lett 393:51-57. doi:10.1016/ j.cplett.2004.06.011

29. Champagne B, Botek E, Nakano M, Nitta T, Yamaguchi K (2005) Basis set and electron correlation effects on the polarizability and second hyperpolarizability of model open-shell pi-conjugated systems. J Chem Phys 122(114315):1-12. doi:10.1063/1.1880992

30. Zhong RL, Xu HL, Sun SL, Qiu YQ, Su ZM (2012) The excess electron in a boron nitride nanotube: pyramidal NBO charge distribution and remarkable first hyperpolarizability. Chem Eur J 18:11350-11355. doi:10.1002/chem.201201570

31. Marques MAL, Rubio A (2009) Time-dependent density-functional theory. Phys Chem Chem Phys 11:4436-4436. doi:10.1039/ B908105B

32. Stratmann RE, Scuseria GE, Frisch MJ (1998) An efficient implementation of time-dependent density-functional theory for the calculation of excitation energies of large molecules. J Chem Phys 109:8218-8224. doi:10.1063/1.477483

33. Ciofini I, Lainé PP, Bedioui F, Adamo C (2004) Photoinduced intramolecular electron transfer in ruthenium and osmium polyads: insights from theory. J Am Chem Soc 126:10763-10777. doi:10.1021/ja0482278

34. Frisch MJ et al. (2010) Gaussian 09W, revision A.02. Gaussian, Inc, Wallingford

35. Gorelsky SI AOMix: Program for molecular orbital analysis, http://www.sg-chem.net/, University of Ottawa, Version 6.5, 2011

36. Gorelsky SI, Lever ABP (2001) Electronic structure and spectra of ruthenium diimine complexes Electronic structure and spectra of ruthenium diimine complexes by density functional theory and INDO/S. Comparison of the two methods. J Organomet Chem 635:187-196. doi:10.1016/S0022-328X(01)01079-8

37. Oudar JL (1977) Optical nonlinearities of conjugated molecules. Stilbene derivatives and highly polar aromatic compounds. J Chem Phys 67:446-457. doi:10.1063/1.434888 